BNL $-72264-2004-C P$

ATF-03E

\title{
STELIA-II: Demonstration of Monoenergetic Laser Acceleration
}

W. D. Kimura, * L. P. Campbell, C. E. Dilley, S. C. Gottschalk, and D. C. Quimby STI Optronics, Bellevue, WA 98004-1495, USA

M. Babzien, I. Ben-Zvi, J. C. Gallardo, K. P. Kusche, I. V. Pogorelsky, J. Skaritka, and V. Yakimenko, Brookhaven National Laboratory, Upton, NY 11973, USA

F. Zhou and D. B. Cline, UCLA, Los Angeles, CA 90095, USA

L. C. Steinhauer, Redmond Plasma Physics Laboratory, Redmond, WA 98052, USA R. H. Pantell, Stanford University, CA 94305, USA

March 2004

Proc. of 2003 Particle Accelerator Conference (PAC2003), Portland, Oregon, May 12-16, 2003, pg. 1909-1911 (TPPG048) 


\title{
STELLA-II: DEMONSTRATION OF MONOENERGETIC LASER ACCELERATION
}

\author{
W. D. Kimura, * L. P. Campbell, C. E. Dilley, S. C. Gottschalk, and D. C. Quimby \\ STI Optronics, Bellevue, WA 98004-1495, USA \\ M. Babzien, I. Ben-Zvi, J. C. Gallardo, K. P. Kusche, I. V. Pogorelsky, J. Skaritka, and \\ V. Yakimenko, Brookhaven National Laboratory, Upton, NY 11973, USA \\ F. Zhou and D. B. Cline, UCLA, Los Angeles, CA 90095, USA \\ L. C. Steinhauer, Redmond Plasma Physics Laboratory, Redmond, WA 98052, USA \\ R. H. Pantell, Stanford University, CA 94305, USA
}

\begin{abstract}
Monoenergetic laser acceleration of trapped microbunches has been demonstrated for the first time. An inverse free electron laser (IFEL) is used to create microbunches, which are then accelerated by a second IFEL using a tapered undulator. An adjustable magnetic field chicane is located between the two IFELs and is used to control the phase of the microbunches with respect to the laser field in the second IFEL. The IFELs are driven by a single laser beam from a high peak power $\mathrm{CO}_{2}$ laser. During the experiment, the trapped portion of the microbunch electrons had an energy gain of $>16 \%$ with an energy width of $\sim 0.86 \%$ (full width at halfmaximum).
\end{abstract}

\section{BACKGROUND}

Laser acceleration of relativistic electrons offers the potential for very high acceleration gradients. Staging of these laser accelerator devices, in which the laser acceleration process is repeated many times, is important for eventually reaching a high net energy gain. However, this staging process must also preserve the electron beam (e-beam) quality so that a useful high-energy $e$-beam is produced.

Staging between two laser-driven acceleration devices was first demonstrated during the Staged Electron Laser Acceleration (STELLA) Experiment [1] located at the Brookhaven National Laboratory (BNL) Accelerator Test Facility (ATF). During this experiment an inverse free electron laser (IFEL), driven by a high peak power $\mathrm{CO}_{2}$ laser at a wavelength of $10.6 \mu \mathrm{m}$, was used to modulate the energy of the $e$-beam passing through the device. The $e$-beam was allowed to drift downstream from the IFEL. During this drift, the fast electrons caught up with the slow ones, resulting in bunching of the electrons into a train microbunches. Each microbunch had a length of $<1 \mu \mathrm{m}$ and was separated from the other microbunches by

*Electronic address: wkimura@stioptronics.com the laser wavelength, i.e., $10.6 \mu \mathrm{m}$. Thus, this first IFEL served as a "buncher." It used a permanent-magnet (PM) uniform gap undulator.

The microbunches then entered a second IFEL, which acted as an "accelerator." It used an identical PM undulator as the buncher. Staging of these two laserdriven devices required rephasing the microbunches with the laser field in the accelerator IFEL.

Separate laser beams were used during STELLA to drive the two IFELs. A relatively weak laser beam $(\sim 24$ MW) modulated the electrons by $\approx \pm 0.5 \%$ in the buncher, and a stronger laser beam $(\sim 200 \mathrm{MW})$ accelerated the microbunches in the accelerator. A passive optical delay line was used to control the phase of the laser beam driving the accelerator with respect to the microbunches entering the second device.

With the $\pm 0.5 \%$ buncher modulation, the electrons needed to drift by $2 \mathrm{~m}$ before they optimally bunched. Hence, the accelerator IFEL was located $2 \mathrm{~m}$ downstream from the buncher IFEL.

While successful at demonstrating staging, the accelerated microbunches during STELLA were not truly trapped in the ponderomotive potential well of the laser field in the accelerator IFEL and, hence, the accelerated electrons had a fairly broad energy spread and were not well separated in energy from the other background electrons.

Therefore, the goal of the STELLA-II experiment was to improve on the quality of the staging process by separating the accelerated microbunch electrons from the background electrons and at the same time maintaining a narrow energy spread of the accelerated electrons. This latter characteristic is important for efficient acceleration of the accelerated microbunches in subsequent acceleration stages.

\section{DESCRIPTION OF EXPERIMENT}

STELLA-II improves upon the basic scheme used during STELLA. A diagram of the experiment is shown in Fig. 1. Two IFELs are still used, but a single laser beam is used to drive both devices. This eliminates phase jitter between the two laser beams, which was evident 


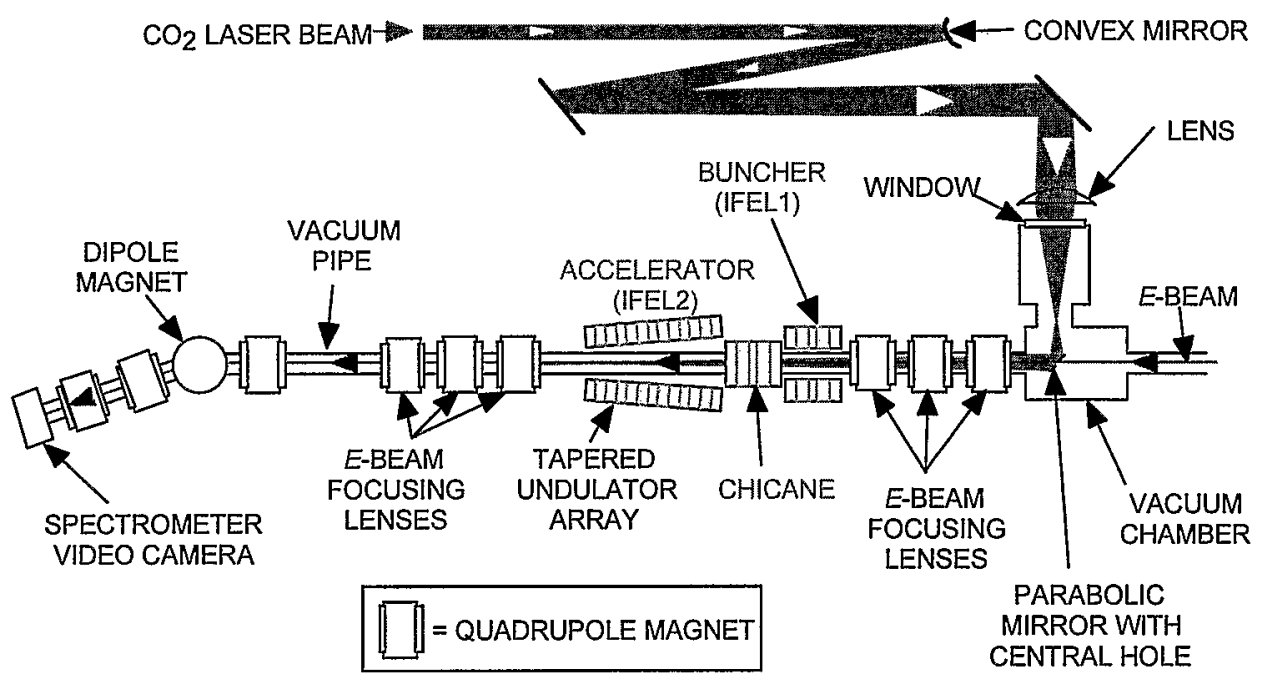

Figure 1. Diagram of STELLA-II experiment.

during the STELLA experiment. In order to use a single laser beam, the two IFELs must also be located close to each other. Therefore, the $2-\mathrm{m}$ drift space between the devices was eliminated and replaced with a magnetic chicane.

The buncher still uses a PM undulator; however, it was redesigned to provide a small amount of modulation when a large amount of laser intensity travels through it since the high-power laser beam driving the accelerator IFEL must also pass through the buncher IFEL.

The chicane is a hybrid permanent magnet/electromagnet (EM) device that makes the modulated electrons bunch at its output by causing the faster electrons to take a shorter path than the slower electrons. The constant PM field provides the nominal curved trajectory for this process while the adjustable EM field allows a fine adjustment on the length of the trajectory. Thus, the EM field serves the same role as the optical delay line during STELLA and provides the phase delay adjustment needed during the experiment.

Another key difference between STELLA-II and STELLA is the usage of a tapered undulator for the accelerator IFEL. STELLA used an untapered undulator; STELLA-II uses an $11 \%$ gap tapered undulator. Using a tapered undulator is critical for achieving higher energy gain, but it requires a minimum amount of laser power to drive the device. Upgrades made to the ATF $\mathrm{CO}_{2}$ laser have greatly increased its available peak power.

The high energy gain provided by a tapered undulator enables the microbunch electrons to be separated away from the unbunched background electrons. As mentioned earlier, this was one of the goals of the STELLA-II experiment.

\section{EXPERIMENTAL RESULTS}

Table 1 lists the basic experimental values during the STELLA-II experiment.
Figure 2 gives an example of the modulation energy spectrum produced by the PM buncher. The chicane is designed to provide optimum bunching when the buncher produces $\pm 0.5 \%$ energy modulation on the $e$-beam. Thus, the modulation seen in Fig. 2 is close to the desired amount. During the experiment it was found there was considerable variation from shot-to-shot of the amount of the modulation and the shape of the modulation spectrum. We believe this may be due to shot-to-shot variations in the laser intensity distribution at the buncher location.

Table 1: STELLA-II experimental parameters.

\begin{tabular}{|l|c|}
\hline \multicolumn{1}{|c|}{ Parameter } & Value \\
\hline$E$-beam energy & $45.6 \mathrm{MeV}$ \\
\hline$E$-beam intrinsic energy spread & $\sim 0.04 \%$ \\
\hline$E$-beam normalized emittance & $\begin{array}{c}1.5 \mathrm{~mm}- \\
\mathrm{mrad}\end{array}$ \\
\hline$E$-beam charge & $\sim 0.1 \mathrm{nC}$ \\
\hline$E$-beam pulse length & $\sim 3 \mathrm{ps}$ \\
\hline Laser wavelength & $10.6 \mu \mathrm{m}$ \\
\hline Laser pulse length & $\sim 180 \mathrm{ps}$ \\
\hline Laser pulse energy & $>5 \mathrm{~J}$ \\
\hline
\end{tabular}

It was also noted during the experiment that the modulation was often considerably larger than the desired $\pm 0.5 \%$. This would lead to overmodulation of the electrons, which meant they would optimally bunch before they entered the accelerator IFEL. 


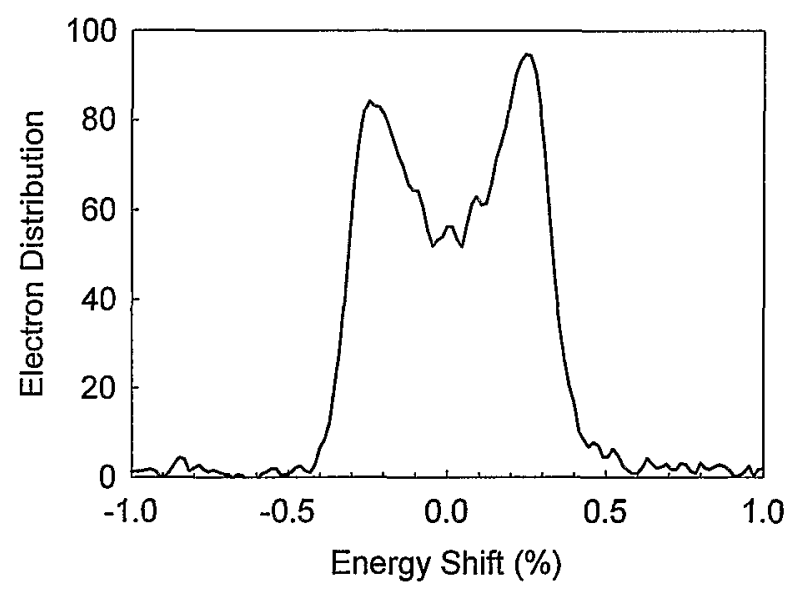

Figure 2: Example of energy modulation spectrum produced by the PM buncher.

In addition, the modulation spectrum did not always display clean double-peaks as seen in Fig. 2, which implied less than ideal modulation. This would lead to less than optimum bunching of the electrons, i.e., the electrons in the microbunches would be spread over a wider phase. We believe this may help explain some of the results seen in energy spectra generated by the tapered undulator.

Figure 3 shows the energy spectrum from the tapered undulator after the electrons pass through the PM buncher and chicane. The EM field on the chicane was adjusted so that the phase delay between the microbunches and the laser field in the accelerator IFEL yielded approximately maximum acceleration.

A narrow spike of accelerated electrons can be clearly seen on the right of the spectrum centered at approximately $16 \%$ energy gain $(>7 \mathrm{MeV}$ net energy gain). The energy width of the peak is $\approx 0.86 \%$ full-widthat-half-maximum (FWHM). The amount of acceleration and width of the peak is consistent with our model simulations for this experiment [2]-[3].

The apparent sharp dips in the spectrum (indicated in red) located at roughly $1 \%$ and $14 \%$ energy shift are due to fiducial marks on the spectrometer phosphor screen that are seen by the video camera. We believe the actual electron energy spectrum continues across these dips in a smooth fashion.

There are electrons in the middle of the spectrum between the narrow peak on the right and the smaller peak on the left. The model predicts with optimal bunching that there should be insignificant electrons between these two peaks. The presence of these electrons between the peaks may be evidence of less than optimal bunching occurring. In other words some of the electrons in the microbunch may be spread out in phase and, therefore, are not trapped as well as the electrons in the right-most peak.

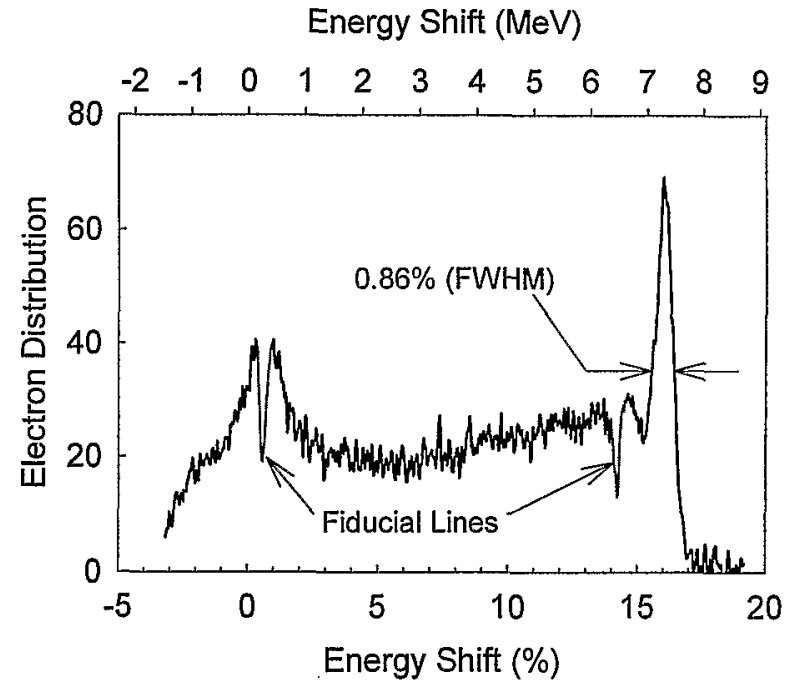

Figure 3: Example of energy modulation spectrum produced by the accelerator IFEL. The fiducial lines indicated in the figure are lines that are on the spectrometer phosphor screen seen by the video camera.

\section{CONCLUSION}

Monoenergetic laser acceleration has been demonstrated for the first time. Trapped electrons in microbunches produced by an IFEL were accelerated by $16 \%$ representing an energy gradient of $>22 \mathrm{MeV} / \mathrm{m}$. The energy width of these trapped electrons is $\sim 0.86 \%$, which is consistent with model predictions.

This accomplishment is an important step towards the goal of eventually realizing practical accelerators based upon laser acceleration.

\section{ACKNOWLEDGEMENTS}

This work was supported by the U.S. Department of Energy, Grant Nos. DE-FG03-98ER41061, DE-AC0298CH10886, and DE-FG03-92ER40695

\section{REFERENCES}

[1] W. D. Kimura, et al., Phys. Rev. Lett. 86, 4041-4043 (2001).

[2] W. D. Kimura, et al., Phys. Rev. ST Accel. Beams 4, 101301 (2001).

[3] W. D. Kimura, et al., Advanced Accelerator Concepts, Jun. 23-28, 2002, Mandalay Beach, CA, AIP Conference Proceedings No. 647, C. E. Clayton and P. Muggli, Eds., (American Institute of Physics, New York, 2002), p. 269-277. 